JOURNAL OF

FUNCTION SPACES AND APPLICATIONS

Volume 5, Number 2 (2007), 167-174
(C) 2007, Scientific Horizon http://www.jfsa.net

\title{
On an abstract nonlinear second order integrodifferential equation
}

\author{
M. B. Dhakne and G. B. Lamb
}

(Communicated by Nigel Kalton)

2000 Mathematics Subject Classification. Primary: 45J05, Secondary: 34K30, 47G20.

Keywords and phrases. Fixed point, global existence of solutions, intregrodifferential equation, Lerray-Schauder alternative, nonlinear second order equation.

Abstract. The aim of the present paper is to study the global existence of solutions of nonlinear second order integrodifferential equation in Banach space. Our analysis is based on an application of the Leray-Schauder alternative and rely on a priori bounds of solutions..

\section{Introduction}

Let $X$ be a Banach space with norm \|\| . Let $B=C([0, T], X)$ be the Banach space of all continuous functions from $[0, T]$ into $X$ endowed with supremum norm

$$
\|x\|_{B}=\sup \{\|x(t)\|: t \in[0, T]\} .
$$

The purpose of this paper is to prove the global existence of solutions of the following initial value problem for second order integrodifferentioal equations of the form

$$
\begin{aligned}
\left(r(t) x^{\prime}(t)\right)^{\prime} & =f\left(t, x(t), \int_{0}^{t} k(t, s) g(s, x(s)) d s\right), t \in[0, T] \\
x(0) & =x_{0}, \quad x^{\prime}(0)=0
\end{aligned}
$$


where $r(t)$ is real-valued positive and sufficiently smooth function defined on $[0, T], f:[0, T] \times X \times X \rightarrow X, g:[0, T] \times X \rightarrow X, k:[0, T] \times[0, T] \rightarrow R$ are continuous functions and $x_{0}$ is a given element of $X$.

Let $x \in L^{1}(0, T ; X)$. By a solution of the initial value problem (1.1) (1.2), we mean the function $x \in C([0, T] ; X)$ given by

$$
x(t)=x_{0}+\int_{0}^{t} \frac{1}{r(s)} \int_{0}^{s} f\left(\tau, x(\tau), \int_{0}^{\tau} k(\tau, \eta) g(\eta, x(\eta)) d \eta\right) d \tau d s,
$$

$t \in[0, T]$.

The main purpose of this paper is to study the global existence of solutions of initial value problem (1.1)-(1.2) by using an application of the topological transversality theorem known as Leray-Schauder alternative. The interesting and useful aspect of the method employed here is that it yields simultaneously the existence of a solution and the maximal interval of existence. Our work is motivated by interesting results obtained by W.F. Trench $[4,5]$ and influenced by the work of the authors [1] and D.B. Pachpatte [3].

\section{Statement of result}

Our Theorem is based on the following theorem, which is a version of the topological transversality theorem given in J. Dugundji and A. Granas [2, p. 61]

Theorem G. Let $Y$ be a convex subset of a normed linear space $E$ and assume $0 \in Y$. Let $F: Y \rightarrow Y$ be a completely continuous operator and let $U(F)=\{x \in Y: x=\lambda F x$ for some $0<\lambda<1\}$. Then either $U(F)$ is unbounded or $F$ has a fixed point.

For convenience, we list the following hypotheses used in our subsequent discussion.

$\left(H_{1}\right)$ There exists a constant $L>0$ such that

$$
\|g(t, x(t))\| \leq L H(\|x(t)\|)
$$

for every $t \in[0, T]$, and $x \in X$, where $H: R_{+} \rightarrow(0, \infty)$ is continuous nondecreasing function.

$\left(H_{2}\right)$ There exists a continuous function $p:[0, T] \rightarrow R_{+}$such that

$$
\|f(t, x, y)\| \leq p(t)(\|x\|+\|y\|),
$$

for every $t \in[0, T]$, and $x, y \in X$. 
$\left(H_{3}\right)$ For each $t \in[0, T]$, the function $g(t,):.[0, T] \times X \rightarrow X$ is continuous and for each $x \in X$ the function $g(., x):[0, T] \times X \rightarrow X$ is strongly measurable.

$\left(H_{4}\right)$ For each $t \in[0, T]$, the function $f(t, .,):.[0, T] \times X \times X \rightarrow X$ is continuous and for each $x, y \in X$ the function $f(., x, y):[0, T] \times$ $X \times X \rightarrow X$ is strongly measurable.

$\left(H_{5}\right)$ For every positive integer $m$ there exists $\alpha_{m} \in L^{1}(0, T)$ such that $\|f(t, x, y)\| \leq \alpha_{m}(t)$ for x,y satisfying $\|x\| \leq m,\|y\| \leq m$ and for a.e. $t \in[0, T]$.

We next establish the following Theorem which deals with the global existence of solution of initial value problem (1.1)-(1.2).

Theorem. Suppose that the hypotheses $\left(H_{1}\right)-\left(H_{5}\right)$ hold. If $T$ satisfies the following condition

$$
\int_{0}^{T} N(s) d s<\int_{c}^{\infty} \frac{d s}{s+M^{*} H(s) T}
$$

where $N(t)=\frac{1}{r(t)} \int_{0}^{t} p(s) d s, c=\left\|x_{0}\right\|, M^{*}=L_{1} L$ and $L_{1}>0$ defined by (3.2), then the initial value problem (1.1)-(1.2) has a solution $x$ defined on $[0, T]$.

\section{Proof of result}

In order to prove the Theorem we apply Theorem G. First we establish the priori bounds for a solution to the initial value problem $(1.1)_{\lambda}-(1.2), \lambda \in$ $(0,1)$ where

$$
\left(r(t) x^{\prime}(t)\right)^{\prime}=\lambda f\left(t, x(t), \int_{0}^{t} k(t, s) g(s, x(s)) d s\right), t \in[0, T] .
$$

Let $x(t)$ be a solution of $(1.1)_{\lambda}-(1.2)$. Then it satisfies the equivalent integral equation

$$
x(t)=x_{0}+\lambda \int_{0}^{t} \frac{1}{r(s)} \int_{0}^{s} f\left(\tau, x(\tau), \int_{0}^{\tau} k(\tau, \eta) g(\eta, x(\eta)) d \eta\right) d \tau d s
$$

where $t \in[0, T]$. Since $k$ is continuous on a compact set $[0, T] \times[0, T]$, there exists $L_{1}>0$ such that

$$
|k(t, s)| \leq L_{1} \quad \text { for } 0 \leq s \leq t \leq T .
$$


Using (3.1),(3.2), hypotheses $\left(H_{1}\right),\left(H_{2}\right)$ and the fact that $\lambda \in(0,1)$, we obtain

$$
\begin{aligned}
\|x(t)\| \leq & \left\|x_{0}\right\|+\int_{0}^{t} \frac{1}{r(s)} \int_{0}^{s} p(\tau)[\|x(\tau)\| \\
& \left.+\left\|\int_{0}^{\tau} k(\tau, \eta) g(\eta, x(\eta)) d \eta\right\|\right] d \tau d s \\
\leq & \left\|x_{0}\right\|+\int_{0}^{t} \frac{1}{r(s)} \int_{0}^{s} p(\tau)[\|x(\tau)\| \\
& \left.+\int_{0}^{\tau} L_{1} L H(\|x(\eta)\|) d \eta\right] d \tau d s
\end{aligned}
$$

Define a function $u(t)$ by the right hand side of (3.3). By using the fact that $H$ is continuous non-decreasing function, we get $\|x(t)\| \leq u(t), u(0)=$ $\left\|x_{0}\right\|=c$ and

$$
u(t) \leq\left\|x_{0}\right\|+\int_{0}^{t} \frac{1}{r(s)} \int_{0}^{s} p(\tau)\left[u(\tau)+\int_{0}^{\tau} L_{1} L H(u(\eta)) d \eta\right] d \tau d s
$$

Clearly $u(t)$ is an increasing function. Denote by $v(t)$ the right hand side of $(3.4)$, then $u(t) \leq v(t), v(0)=\left\|x_{0}\right\|=c$ and

$$
v(t)=\left\|x_{0}\right\|+\int_{0}^{t} \frac{1}{r(s)} \int_{0}^{s} p(\tau)\left[u(\tau)+\int_{0}^{\tau} L_{1} L H(u(\eta)) d \eta\right] d \tau d s
$$

It is clear that $v(t)$ is also an increasing function. Differentiating, we have

$$
v^{\prime}(t) \leq\left\{v(t)+M^{*} H(v(t)) T\right\} \frac{1}{r(t)} \int_{0}^{t} p(s) d s .
$$

Hence

$$
\frac{v^{\prime}(t)}{v(t)+M^{*} H(v(t)) T} \leq N(t)
$$

Integrating (3.6) from 0 to $t$ and by making use of the change of variables $t \rightarrow s=v(t)$ and the condition (2.1), we get

$$
\int_{c}^{v(t)} \frac{d s}{s+M^{*} H(s) T} \leq \int_{0}^{t} N(s) d s \leq \int_{0}^{T} N(s) d s<\int_{c}^{\infty} \frac{d s}{s+M^{*} H(s) T} .
$$

From (3.7) we conclude by the mean value theorem that there is a constant $Q$ independent of $\lambda \in(0,1)$ such that $v(t) \leq Q$ for $t \in[0, T]$. Then we 
have successively $u(t) \leq Q,\|x(t)\| \leq Q$ for $t \in[0, T]$ and consequently

$$
\|x\|_{B}=\sup \{\|x(t)\|: t \in[0, T]\} \leq Q .
$$

Now, we rewrite the problem (1.1)-(1.2) as follows: If $y \in B$ and $x(t)$ defined by $x(t)=x_{0}+y(t), t \in[0, T]$, then it is easy to observe that $y(t)$ satisfies an integral equation

$$
y(t)=\int_{0}^{t} \frac{1}{r(s)} \int_{0}^{s} f\left(\tau, y(\tau)+x_{0}, \int_{0}^{\tau} k(\tau, \eta) g\left(\eta, y(\eta)+x_{0}\right) d \eta\right) d \tau d s,
$$

if and only if $x(t)$ satisfies IVP (1.1)-(1.2) or their equivalent integral equation

$$
x(t)=x_{0}+\int_{0}^{t} \frac{1}{r(s)} \int_{0}^{s} f\left(\tau, x(\tau), \int_{0}^{\tau} k(\tau, \eta) g(\eta, x(\eta)) d \eta\right) d \tau d s .
$$

Define $F: B_{0} \rightarrow B_{0}, B_{0}=\{y \in B: y(0)=0\}$ by

$$
(F y)(t)=\int_{0}^{t} \frac{1}{r(s)} \int_{0}^{s} f\left(\tau, y(\tau)+x_{0}, \int_{0}^{\tau} k(\tau, \eta) g\left(\eta, y(\eta)+x_{0}\right) d \eta\right) d \tau d s
$$

where $t \in[0, T]$.

Now, we prove that $F: B_{0} \rightarrow B_{0}$ is continuous. Let $\left\{y_{n}\right\}$ be a sequence of elements of $B_{0}$ converging to $y$ in $B_{0}$. Then by using $\left(H_{3}\right)$ and $\left(H_{4}\right)$, we get

$$
\begin{aligned}
f\left(s, y_{n}(s)+x_{0}, \int_{0}^{s} k(s, \tau) g\left(\tau, y_{n}(\tau)+x_{0}\right) d \tau\right) \\
\rightarrow f\left(s, y(s)+x_{0}, \int_{0}^{s} k(s, \tau) g\left(\tau, y(\tau)+x_{0}\right) d \tau\right)
\end{aligned}
$$

for each $t \in[0, T]$. From equation (3.8), we get

$\left(F y_{n}\right)(t)=\int_{0}^{t} \frac{1}{r(s)} \int_{0}^{s} f\left(\tau, y_{n}(\tau)+x_{0}, \int_{0}^{\tau} k(\tau, \eta) g\left(\eta, y_{n}(\eta)+x_{0}\right) d \eta\right) d \tau d s$

Now, $\left\|F y_{n}-F y\right\|_{B}=\sup _{0 \leq t \leq T}\left\|\left(F y_{n}\right)(t)-(F y)(t)\right\|$. By using hypotheses $\left(H_{3}\right)$ and $\left(H_{4}\right)$ and the dominated convergence theorem, we have 


$$
\begin{aligned}
\| & \left(F y_{n}\right)(t)-(F y)(t) \| \\
\leq & \int_{0}^{t} \frac{1}{r(s)} \int_{0}^{s} \| f\left(\tau, y_{n}(\tau)+x_{0}, \int_{0}^{\tau} k(\tau, \eta) g\left(\eta, y_{n}(\eta)+x_{0}\right) d \eta\right) \\
& -f\left(\tau, y(\tau)+x_{0}, \int_{0}^{\tau} k(\tau, \eta) g\left(\eta, y(\eta)+x_{0}\right) d \eta\right) \| d \tau d s \\
\rightarrow & \text { as } n \rightarrow \infty
\end{aligned}
$$

and it follows that $\left\|F y_{n}-F y\right\|_{B} \rightarrow 0$ as $n \rightarrow \infty$ i.e. $F y_{n} \rightarrow F y$ in $B_{0}$ as $y_{n} \rightarrow y$ in $B_{0}$. Thus $F$ is continuous.

Now, we prove that $F$ maps a bounded set of $B_{0}$ into a precompact set in $B_{0}$. Let $B_{m}=\left\{y \in B_{0}:\|y\|_{B} \leq m\right\}$ for $m \geq 1$. We first show that $F$ maps $B_{m}$ into an equicontinuous family of functions with values in $X$. Let $y \in B_{m}, t_{1}, t_{2} \in[0, T]$ and $0 \leq t_{1} \leq t_{2} \leq T$. Using (3.2),hypotheses $\left(H_{1}\right),\left(H_{2}\right)$ and condition $(2.1)$, we have

$$
\begin{aligned}
& \left\|(F y)\left(t_{1}\right)-(F y)\left(t_{2}\right)\right\| \\
& \quad \leq \int_{t_{1}}^{t_{2}} \frac{1}{r(s)} \int_{0}^{s}\left\|f\left(\tau, y(\tau)+x_{0}, \int_{0}^{\tau} k(\tau, \eta) g\left(\eta, y(\eta)+x_{0}\right) d \eta\right)\right\| d \tau d s \\
& \quad \leq \int_{t_{1}}^{t_{2}} \frac{1}{r(s)} \int_{0}^{s} p(\tau)\left[m+c+\int_{0}^{\tau} L_{1} L H(m+c) d \eta\right] d \tau d s \\
& \quad \leq\left[m+c+M^{*} H(m+c) T\right] \int_{t_{1}}^{t_{2}} N(s) d s .
\end{aligned}
$$

The right hand side of (3.10) tends to zero as $t_{2}-t_{1} \rightarrow 0$. Thus $F_{B_{m}}$ is an equicontinuous family of functions with values in $X$.

We next show that $F_{B_{m}}$ is uniformly bounded. From the equation (3.8) and using $(3.2)$, hypotheses $\left(H_{1}\right),\left(H_{2}\right)$, the condition (2.1) and the fact that $\|y\|_{B} \leq m$, we have

$$
\begin{aligned}
& \|(F y)(t \| \\
& \leq \int_{0}^{t} \frac{1}{r(s)} \int_{0}^{s}\left\|f\left(\tau, y(\tau)+x_{0}, \int_{0}^{\tau} k(\tau, \eta) g\left(\eta, y(\eta)+x_{0}\right) d \eta\right)\right\| d \tau d s \\
& \leq \int_{0}^{t} \frac{1}{r(s)} \int_{0}^{s} p(\tau)\left[m+c+\int_{0}^{\tau} L_{1} L H(m+c) d \eta\right] d \tau d s \\
& \leq\left[m+c+M^{*} H(m+c) T\right] \int_{0}^{t} N(s) d s
\end{aligned}
$$




$$
\leq\left[m+c+M^{*} H(m+c) T\right] \int_{0}^{T} N(s) d s .
$$

This yields that the set $\left\{(F y)(t):\|y\|_{B} \leq m, 0 \leq t \leq T\right\}$ is bounded in $X$ and hence, $\left\{F_{B_{m}}\right\}$ is uniformly bounded.

We have already shown that $F_{B_{m}}$ is an equicontinuous and uniformly bounded collection. To prove the set $F_{B_{m}}$ is precompact in $B$, it is sufficient, by Arzela-Ascoli's argument, to show that the set $\{(F y)(t): y \in$ $\left.B_{m}\right\}$ is precompact in $X$ for each $t \in[0, T]$. Since $(F y)(0)=0$ for $y \in B_{m}$, it suffices to show this for $0<t \leq T$. Let $0<t \leq T$ be fixed and $\epsilon$ a real number satisfying $0<\epsilon<t$. For $y \in B_{m}$, we define

$$
\begin{aligned}
\left(F_{\epsilon} y\right)(t)= & \int_{0}^{t-\epsilon} \frac{1}{r(s)} \int_{0}^{s} f\left(\tau, y(\tau)+x_{0}\right. \\
& \left.\int_{0}^{\tau} k(\tau, \eta) g\left(\eta, y(\eta)+x_{0}\right) d \eta\right) d \tau d s
\end{aligned}
$$

The set $y_{\epsilon}(t)=\left\{\left(F_{\epsilon} y\right)(t): y \in B_{m}\right\}$ is precompact in $X$ for every $\epsilon, 0<\epsilon<t$. Moreover for every $y \in B_{m}$, we get

$$
\begin{aligned}
& (F y)(t)-\left(F_{\epsilon} y\right)(t) \\
& =\int_{t-\epsilon}^{t} \frac{1}{r(s)} \int_{0}^{s} f\left(\tau, y(\tau)+x_{0}, \int_{0}^{\tau} k(\tau, \eta) g\left(\eta, y(\eta)+x_{0}\right) d \eta\right) d \tau d s
\end{aligned}
$$

By making use of $(3.2)$, hypotheses $\left(H_{1}\right),\left(H_{2}\right)$, the condition $(2.1)$ and the fact that $\|y(s)\| \leq m$, we have

$$
\begin{aligned}
& \left\|(F y)(t)-\left(F_{\epsilon} y\right)(t)\right\| \\
& \leq \int_{t-\epsilon}^{t} \frac{1}{r(s)} \int_{0}^{s} p(\tau)\left[\left\|y(\tau)+x_{0}\right\|+\left\|\int_{0}^{\tau} k(\tau, \eta) g\left(\eta, y(\eta)+x_{0}\right) d \eta\right\|\right] d \tau d s \\
& \leq \int_{t-\epsilon}^{t} \frac{1}{r(s)} \int_{0}^{s} p(\tau)\left[\|y(\tau)\|+\left\|x_{0}\right\|+\int_{0}^{\tau} L_{1} L H\left(\|y(\eta)\|+\left\|x_{0}\right\|\right) d \eta\right] d \tau d s \\
& \leq \int_{t-\epsilon}^{t} \frac{1}{r(s)} \int_{0}^{s} p(\tau)\left[m+c+\int_{0}^{\tau} L_{1} L H(m+c) d \eta\right] d \tau d s \\
& \leq\left[m+c+M^{*} H(m+c) T\right] \int_{t-\epsilon}^{t} N(s) d s
\end{aligned}
$$


This implies that there exist precompact sets arbitrarily close to the set $\left\{(F y)(t): y \in B_{m}\right\}$. Hence the set $\left\{(F y)(t): y \in B_{m}\right\}$ is precompact in $X$. Thus we have shown that $F$ is completely continuous operator.

Moreover, the set

$$
U(F)=\left\{y \in B_{0}: y=\lambda F y, \lambda \in(0,1)\right\},
$$

is bounded in $B$, since for every $y$ in $U(F)$, the function $x(t)=y(t)+x_{0}$ is a solution of $(1.1)_{\lambda}-(1.2)$ for which we have proved $\|x\|_{B} \leq Q$ and hence $\|y\|_{B} \leq Q+c$. Now, by virtue of Theorem $\mathrm{G}$, the operator $F$ has a fixed point in $B_{0}$. Therefore, the initial value problem $(1.1)-(1.2)$ has a solution. Thus the proof of the Theorem is complete.

Acknowledgement. Authors are grateful to the referee for his/her comments and suggestions.

\section{References}

[1] M.B. Dhanke and G.B. Lamb, Existence result for an abstract nonlinear integrodifferential equation, Ganit: J. Bangladesh Math. Soc., 21 (2001), 29-37.

[2] J. Dugundji and A. Granas, Fixed Point Theory I, Monografie Matematyczne, PWN, WARSAW, 1982.

[3] D.B. Pachpatte, Existence theorems for some integrodifferential equations, Libertas Math., XV (1995), 189-202.

[4] W.F. Trench, Functional perturbations of second order differential equations, SIAM J. Math. Anal., 16 (4) (1985), 741-756.

[5] W.F. Trench, Linear perturbations of a nonoscillatory second order equation, Proc. Amer. Math. Soc., 97 (3) (1986), 423-428.

Department of Mathematics

Dr. Babasaheb Ambedkar Marathwada University

Aurangabad-431004 (M.S.)

India

(E-mail : mbdhakne@yahoo.com)

Department of Mathematics

Baliram Patil Arts, Commerce and Science College

Kinwat-431804 (M.S.)

India

(Received : February 2005) 


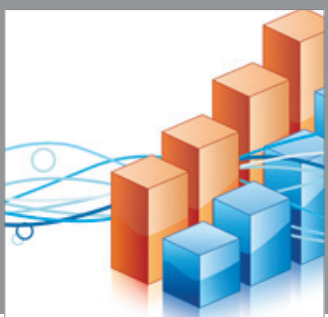

Advances in

Operations Research

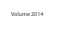

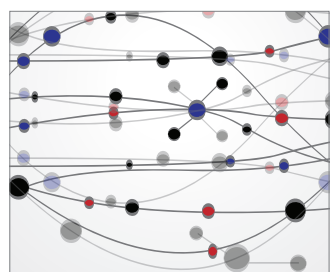

\section{The Scientific} World Journal
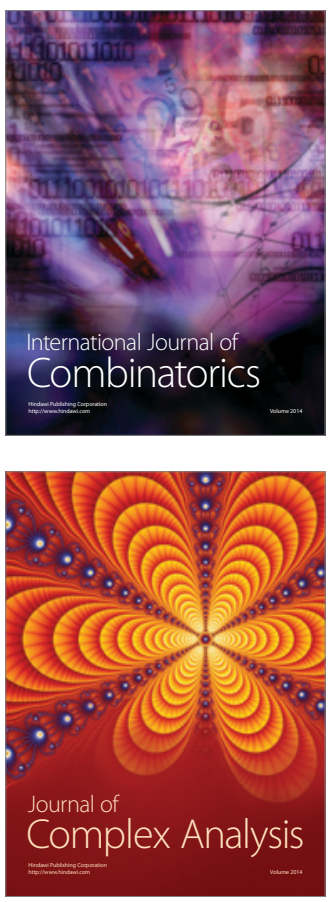

International Journal of

Mathematics and

Mathematical

Sciences
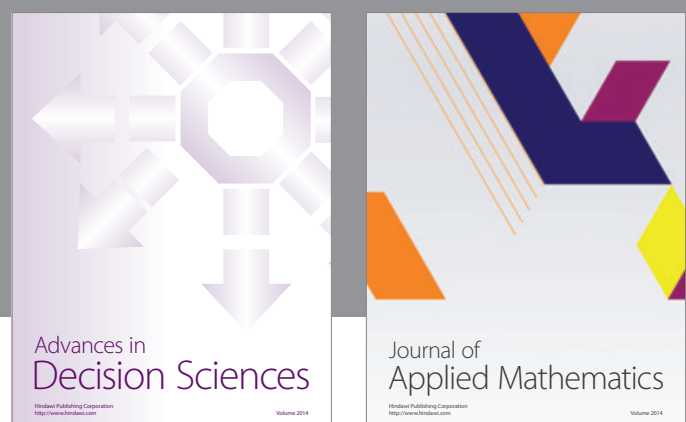

Journal of

Applied Mathematics
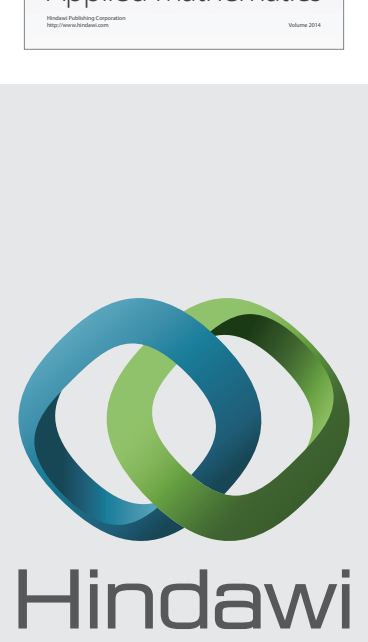

Submit your manuscripts at http://www.hindawi.com
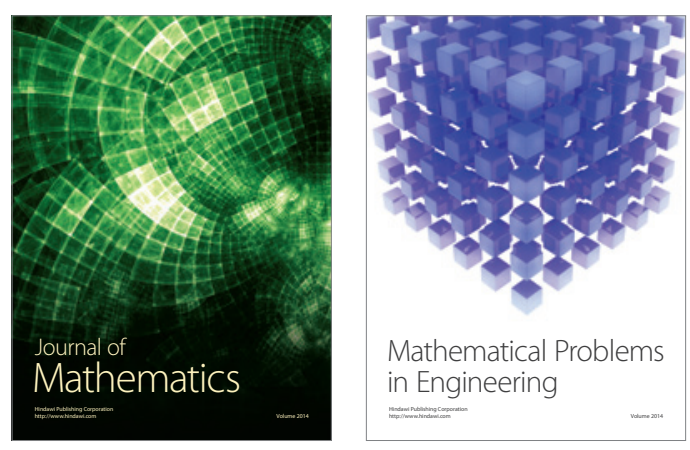

Mathematical Problems in Engineering
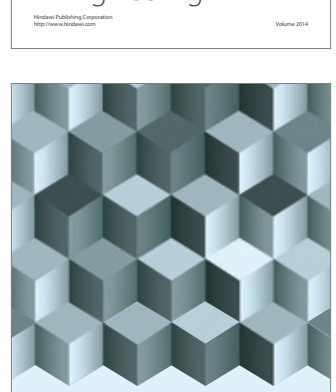

Journal of

Function Spaces
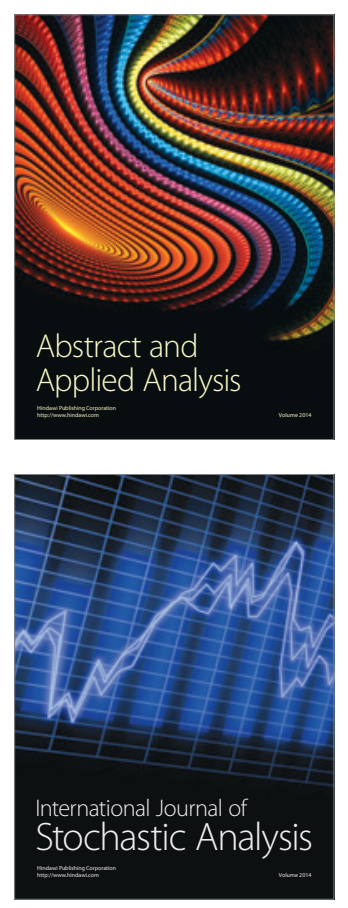

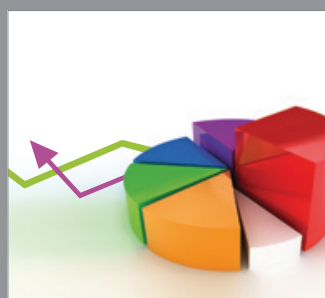

ournal of

Probability and Statistics

Promensencen
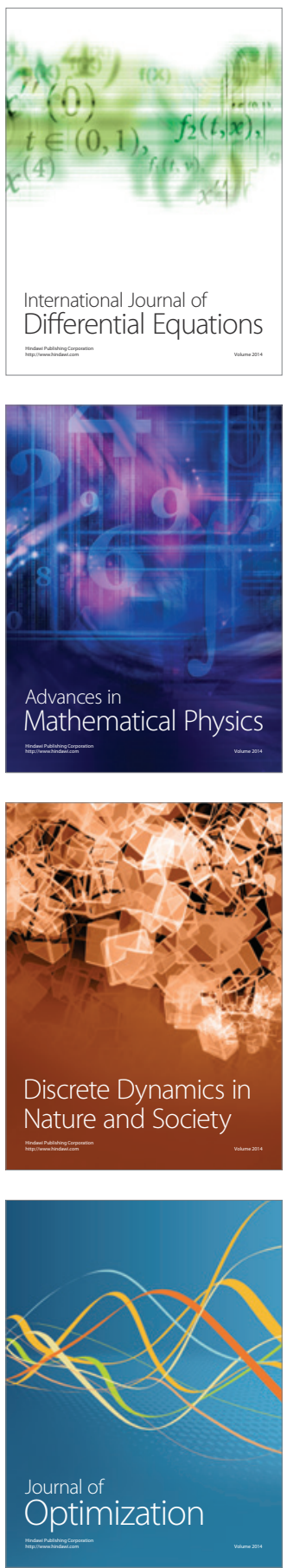